Pacific

Journal of

Mathematics

THE ANOSOV THEOREM FOR INFRANILMANIFOLDS WITH CYCLIC HOLONOMY GROUP

KAREl DeKimpe, BRAm De Rock AND Wim Malfait 


\title{
THE ANOSOV THEOREM FOR INFRANILMANIFOLDS WITH CYCLIC HOLONOMY GROUP
}

\author{
Karel Dekimpe, Bram De Rock And Wim Malfait
}

\begin{abstract}
A celebrated theorem of Anosov states that for any continuous self-map $f: M \rightarrow M$ of a nilmanifold $M$, the Nielsen number equals the Lefschetz number in absolute value. Anosov also showed that this result does not hold for infranilmanifolds, even in the simplest possible situation of flat manifolds with cyclic holonomy group.

Nevertheless, in this paper we extend Anosov's theorem to infranilmanifolds with cyclic holonomy group, provided a certain easily checked condition on the holonomy representation is satisfied.

In the case of flat manifolds with cyclic holonomy group this condition is necessary and sufficient. In the general case of all infranilmanifolds with cyclic holonomy group, we provide an example which shows that this condition is no longer necessary.

We also prove that for any nonorientable flat manifold Anosov's theorem is not true, but again the same example shows that this is not valid in general for nonorientable infranilmanifolds.
\end{abstract}

\section{Introduction}

Let $M$ be a smooth closed manifold and let $f: M \rightarrow M$ be a continuous self-map of $M$. One of the main objectives in fixed point theory is to calculate $M F(f)$, the minimum number of fixed points among all maps homotopic to $f$. Unfortunately this number is not readily computable, since one needs to construct all maps homotopic to $f$. Therefore other numbers are associated to $f$ which can provide information on $M F(f)$.

Two of these numbers are the Lefschetz number $L(f)$ and the Nielsen number $N(f)$; see Section 2 for the definitions. The Nielsen number is a lower bound for $M F(f)$ and F. Wecken [1942] and B. Jiang [1993] proved that this lower bound is sharp for all compact, connected manifolds except for surfaces of negative Euler characteristic. In this paper we focus on infranilmanifolds and so, by the result just mentioned, for these manifolds, $N(f)$ is now a main objective. Unfortunately with $N(f)$ one faces the same problem as with $M F(f)$ : it is not readily computable.

MSC2000: 55M20, 37C25.

Keywords: Nielsen number, Lefschetz number, infranilmanifolds, fixed point theory. 
The Lefschetz number, on the other hand, is easier to compute, but now the problem is that in general $L(f)$ is not very informative. Therefore a considerable amount of work has been done on investigating the relation between $L(f)$ and $N(f)$. In this respect, D. Anosov [1985] proved that $N(f)=|L(f)|$ for all continuous maps $f: M \rightarrow M$ if $M$ is a nilmanifold. He also showed that this result does not hold for all infranilmanifolds (a nilmanifold can be seen as an infranilmanifold), since he constructed a continuous map $f: K \rightarrow K$ of the Klein bottle $K$ (which is a flat manifold having $\mathbb{Z}_{2}$ as holonomy group) such that $N(f) \neq|L(f)|$.

In the literature one can find extensions of this result of Anosov to other classes of manifolds. For instance, Keppelmann and McCord [1995] showed that for continuous maps of exponential solvmanifolds the same relation holds. The authors did the same for maps of orientable generalized Hantzsche-Wendt manifolds [Dekimpe et al. 2004] and infranilmanifolds with an abelian holonomy group of odd order [2006].

From the observations of Anosov on the Klein bottle, we learn that in general his theorem does not hold for infranilmanifolds with cyclic holonomy group $F$. However, in Section 3 we establish a sufficient condition on the holonomy representation of $F$ such that Anosov's theorem still holds for these manifolds. More concretely, suppose that $x_{0}$ generates the holonomy group $F$ and $T: F \rightarrow$ Aut $G$ is the associated holonomy representation (where $G$ is the covering Lie group). We show that Anosov's theorem still holds if -1 is not an eigenvalue of $T_{*}\left(x_{0}\right)$. This is therefore a sufficient condition and so we extended Anosov's theorem to a new (and large) class of infranilmanifolds.

In subsequent sections we examine whether this condition is also necessary and we need to distinguish two cases. In Section 4 we restrict ourselves to flat manifolds with cyclic holonomy group. In that case we are able to show that the sufficient condition is also necessary, so for flat manifolds with cyclic holonomy group the problem is completely solved. The techniques used in the proof of this result are extended to show that Anosov's theorem is not true for any nonorientable flat manifold. So we also conclude that for flat manifolds, orientability is a necessary condition for the extension of Anosov's theorem.

In Section 5 we present a nonorientable infranilmanifold $M_{1}$ with cyclic holonomy group $F_{1}$ which does not satisfy the sufficient condition mentioned above. Nevertheless, for any continuous map $f: M_{1} \rightarrow M_{1}$, we do have $N(f)=|L(f)|$, so Anosov's theorem holds for $M_{1}$. This shows that the results obtained in Section 4 no longer hold in general for infranilmanifolds, illustrating that the situation for infranilmanifolds is more complicated than for flat manifolds. 


\section{Preliminaries}

Let $G$ be a connected, simply connected, nilpotent Lie group. An affine endomorphism of $G$ is an element $(\delta, \mathfrak{D})$ of the semigroup $G \rtimes$ Endo $G$, with $\delta$ (the translational part) an element of $G$ and $\mathfrak{D}$ (the linear part) an element of Endo $G$, the semigroup of all endomorphisms of $G$. The product of two affine endomorphisms is given by $(\delta, \mathfrak{D})\left(\delta^{\prime}, \mathfrak{D}^{\prime}\right)=\left(\delta \cdot \mathfrak{D}\left(\delta^{\prime}\right), \mathfrak{D} \mathfrak{D}^{\prime}\right)$ and $(\delta, \mathfrak{D})$ maps an element $x \in G$ to $\delta \cdot \mathfrak{D}(x)$. If the linear part $\mathfrak{D}$ belongs to Aut $G$, then $(\delta, \mathfrak{D})$ is an invertible affine transformation of $G$. We write Aff $G=G \rtimes$ Aut $G$ for the group of invertible affine transformations of $G$.

Infranilmanifolds and continuous maps. We quickly recall the notion of almostcrystallographic groups and infranilmanifolds. See [Dekimpe 1996] for details.

An almost-crystallographic group is a subgroup $E$ of $\operatorname{Aff} G$, such that its subgroup of pure translations $N=E \cap G$ is a uniform lattice of $G$ and that $N$ is of finite index in $E$. Therefore the quotient group $F=E / N$ is finite; $F$ is called the holonomy group of $E$, and is isomorphic to the image of $E$ under the natural projection Aff $G \rightarrow$ Aut $G$, and hence can be viewed as a subgroup of Aut $G$ and of Aff $G$.

An almost-crystallographic group acts properly discontinuously on (the corresponding) $G$ and the orbit space $E \backslash G$ is compact. When $E$ is a torsion-free almost-crystallographic group, it is referred to as an almost-Bieberbach group and the orbit space $M=E \backslash G$ is called an infranilmanifold. In this case $E$ equals the fundamental group $\pi_{1}(M)$ of the infranilmanifold, and $F$ is referred to as the holonomy group of $M$. In the special case that $G$ is abelian (that is, $G=\mathbb{R}^{n}$ ), $M$ is a flat manifold.

As indicated above, any almost-crystallographic group determines a faithful representation $T: F \rightarrow$ Aut $G$, called the holonomy representation, which is induced by the natural projection $E \rightarrow$ Aut $G$ - where we regard $F$ as a subgroup of Aut $G$. Let $\mathfrak{g}$ denote the Lie algebra of $G$. By taking differentials, the holonomy representation also induces a faithful representation

$$
\begin{aligned}
T_{*}: F & \rightarrow \text { Aut } \mathfrak{g} \\
x & \mapsto T_{*}(x):=d(T(x)) .
\end{aligned}
$$

Theorem 2.1 [Lee 1995]. Let $E, E^{\prime} \subset \operatorname{Aff} G$ be two almost-crystallographic groups. Then for any homomorphism $\theta: E \rightarrow E^{\prime}$, there exists some $g=(\delta, \mathfrak{D})$ in $G \rtimes$ Endo $G$ such that $\theta(\alpha) \cdot g=g \cdot \alpha$ for all $\alpha \in E$.

Corollary 2.2. Let $M=E \backslash G$ be an infranilmanifold and $f: M \rightarrow M$ a continuous map of $M$. Then $f$ is homotopic to a map $h: M \rightarrow M$ induced by an affine endomorphism $(\delta, \mathfrak{D}): G \rightarrow G$. 
We say that $(\delta, \mathfrak{D})$ is a homotopy lift of $f$. Since any map $f: M \rightarrow M$ induces a homomorphism $f_{*}: \pi_{1}(M) \rightarrow \pi_{1}(M)$, one can find a homotopy lift for a given $f$ by using Theorem 2.1 for the homomorphism $f_{*}$ (for details we refer to [Lee 1995, page 161]). Using this method one can actually characterize, up to homotopy, all continuous self-maps of a given infranilmanifold $M$. Indeed, for infranilmanifolds $\pi_{1}(M)$ is finitely generated and therefore one can construct all possible homomorphisms on $\pi_{1}(M)$. So by Theorem 2.1 we then can construct all suitable affine endomorphisms and any continuous map of $M$ is homotopic to an induced map of such a suitable affine endomorphism. See [Dekimpe et al. 2004], for instance, for an application of this method.

The Lefschetz and Nielsen numbers on infranilmanifolds. Let $M$ be a compact manifold and assume $f: M \rightarrow M$ is a continuous map. The Lefschetz number $L(f)$ is defined by

$$
L(f)=\sum_{i}(-1)^{i} \operatorname{Trace}\left(f_{*}: H_{i}(M, \mathbb{Q}) \rightarrow H_{i}(M, \mathbb{Q})\right) .
$$

The set Fix $f$ of fixed points of $f$ is partitioned into equivalence classes, referred to as fixed point classes, by the relation: $x, y \in \operatorname{Fix} f$ are $f$-equivalent if and only if there is a path $w$ from $x$ to $y$ such that $w$ and $f w$ are homotopic (rel endpoints). To each class one assigns an integer index. A fixed point class is said to be essential if its index is nonzero. The Nielsen number of $f$ is the number of essential fixed point classes of $f$. The relation between $L(f)$ and $N(f)$ is given by the property that $L(f)$ is exactly the sum of the indices of all fixed point classes. For details see [Brown 1971; Jiang 1983; Kiang 1989].

In this article we examine the relation $N(f)=|L(f)|$ for continuous maps $f$ : $M \rightarrow M$ on an infranilmanifold $M$. Since $L(f)$ and $N(f)$ are homotopy invariants, one can restrict to those maps that are induced by an affine endomorphism of the covering Lie group $G$.

This is exploited completely in the following theorem of K. B. Lee, which will play a crucial role throughout the paper.

Theorem 2.3 [Lee 1995]. Let $f: M \rightarrow M$ be a continuous map of an infranilmanifold $M$ and let $T: F \rightarrow$ Aut $G$ be the associated holonomy representation. Let $(\delta, \mathfrak{D}) \in G \rtimes$ Endo $G$ be a homotopy lift of $f$.

- $N(f)=L(f)$ if and only if $\operatorname{det}\left(I_{n}-T_{*}(x) \mathfrak{D}_{*}\right) \geq 0$ for all $x \in F$.

- $N(f)=-L(f)$ if and only if $\operatorname{det}\left(I_{n}-T_{*}(x) \mathfrak{D}_{*}\right) \leq 0$ for all $x \in F$.

Based on Theorem 2.3 we described in [Dekimpe et al. 2006] a class of maps on infranilmanifolds for which Anosov's theorem always holds. (We do not claim that such maps exist on all infranilmanifolds.) We recall this result here: 
Proposition 2.4. Let $M$ be an infranilmanifold with holonomy group $F$ and associated holonomy representation $T: F \rightarrow$ Aut $G$. Let $f: M \rightarrow M$ be a continuous map with homotopy lift $(\delta, \mathfrak{D})$.

Suppose that $T_{*}(x) \mathfrak{D}_{*} \neq \mathfrak{D}_{*} T_{*}(x)$ for all $x \in F$ distinct from the identity. Then

$$
\operatorname{det}\left(I_{n}-\mathfrak{D}_{*}\right)=\operatorname{det}\left(I_{n}-T_{*}(x) \mathfrak{D}_{*}\right) \quad \text { for all } x \in F,
$$

and hence $N(f)=|L(f)|$.

Infranilmanifolds with cyclic holonomy group. The results in this paper depend heavily on the fact that we work with infranilmanifolds with cyclic holonomy group. To take advantage of this we need two lemmas concerning matrices of finite order (or cyclic groups of matrices). We leave the proof of these lemmas to the reader. (By a matrix $B$ or an eigenvalue $\lambda$ of order $d$, we mean one such that $B^{d}=I$ or $\lambda^{d}=1$, where $d$ is the smallest positive integer for which this holds.)

Lemma 2.5. Let $B \in \mathrm{GL}(n, \mathbb{R})$ have order $d$ and let $d_{0}, d_{1}, \ldots, d_{t}$ be the divisors of $d$, ordered so that $1=d_{0}<d_{1}<\cdots<d_{t}=d$. Then there exists $n_{0}, n_{1}, \ldots, n_{t} \in \mathbb{N}$ and $a P \in \mathrm{GL}(n, \mathbb{R})$ such that $n_{0}+n_{1}+\cdots+n_{t}=n$ and

$$
P B P^{-1}=\left(\begin{array}{cccc}
B_{0} & 0 & \cdots & 0 \\
0 & B_{1} & \cdots & 0 \\
\vdots & \vdots & \ddots & \vdots \\
0 & 0 & \cdots & B_{t}
\end{array}\right),
$$

with $B_{i} \in \mathrm{GL}\left(n_{i}, \mathbb{R}\right)$ having only eigenvalues of order $d_{i}(0 \leq i \leq t)$.

Note that certain $n_{i}$ from the lemma might be 0 .

Lemma 2.6. Let $d>0$ be an integer with divisors $d_{0}, d_{1}, \ldots, d_{t}$, ordered so that $1=d_{0}<d_{1}<\cdots<d_{t}=d$. Suppose that

$$
B=\left(\begin{array}{cccc}
B_{0} & 0 & \cdots & 0 \\
0 & B_{1} & \cdots & 0 \\
\vdots & \vdots & \ddots & \vdots \\
0 & 0 & \cdots & B_{t}
\end{array}\right),
$$

with $B_{i} \in \operatorname{GL}\left(n_{i}, \mathbb{R}\right)$ having only eigenvalues of order $d_{i}(0 \leq i \leq t)$. Set $n=$ $n_{0}+\cdots+n_{t}$ and suppose $C \in M_{n}(\mathbb{R})$ is such that $C B=B^{l} C$ with $0 \leq l<d$. Then

$$
C=\left(\begin{array}{cccc}
C_{0} & 0 & \cdots & 0 \\
* & C_{1} & \cdots & 0 \\
\vdots & \vdots & \ddots & \vdots \\
* & * & \cdots & C_{t}
\end{array}\right),
$$

where $C_{i} \in M_{n_{i}}(\mathbb{R})$ for $0 \leq i \leq t$ and $*$ indicates any block of real numbers. 
Now suppose that $M$ is an infranilmanifold with cyclic holonomy group $F$ generated by an element $x_{0}$, and that $T: F \rightarrow$ Aut $G$ is the associated holonomy representation. Assume that $F$, and so $x_{0}$, is of order $2^{r} k$ with $r \geq 0$ and $k$ an odd integer. Let $f: M \rightarrow M$ be a continuous map and $(\delta, \mathfrak{D})$ a homotopy lift of $f$.

Because of Theorem 2.1 we know that there exists an integer $l$, with $0 \leq l<2^{r} k$, such that $T_{*}\left(x_{0}^{l}\right) \mathfrak{D}_{*}=\mathfrak{D}_{*} T_{*}\left(x_{0}\right)$. Indeed, if $\tilde{x}_{0} \in E=\pi_{1}(M)$ is a preimage of $x_{0}$, then $x_{0}^{l}$ is the natural projection of $f_{*}\left(\tilde{x}_{0}\right)$, where $f_{*}$ denotes the morphism induced by $f$ on $\pi_{1}(M)$. Therefore we can apply the previous lemmas to $T_{*}\left(x_{0}\right)$ and $\mathfrak{D}_{*}$. Suppose that $d_{0}, d_{1}, \ldots, d_{t}$ are the divisors of $2^{r} k$, with $1=d_{0}<d_{1}<\cdots<d_{t}=2^{r} k$. Because of Lemma 2.5 there exist $n_{0}, n_{1}, \ldots, n_{t} \in \mathbb{N}$ and $P \in \mathrm{GL}(n, \mathbb{R})$ such that $n_{0}+n_{1}+\cdots+n_{t}=n$ and

$$
P T_{*}\left(x_{0}\right) P^{-1}=\left(\begin{array}{cccc}
A_{0} & 0 & \cdots & 0 \\
0 & A_{1} & \cdots & 0 \\
\vdots & \vdots & \ddots & \vdots \\
0 & 0 & \cdots & A_{t}
\end{array}\right),
$$

with $A_{i} \in \mathrm{GL}\left(n_{i}, \mathbb{R}\right)$ having only eigenvalues of order $d_{i}(0 \leq i \leq t)$. Each $d_{i}$ can be written as $2^{s} d$, with $s \geq 0$ and $d$ an odd integer. (Note that since $T_{*}\left(x_{0}\right)$ is of finite order, the only possible eigenvalues are \pm 1 or nonreal. Also note that any $n_{i}$ can be zero.) Because of Lemma 2.6 we then have

$$
P \mathfrak{D}_{*} P^{-1}=\left(\begin{array}{cccc}
D_{0} & 0 & \cdots & 0 \\
* & D_{1} & \cdots & 0 \\
\vdots & \vdots & \ddots & \vdots \\
* & * & \cdots & D_{t}
\end{array}\right),
$$

with $D_{i} \in M_{n_{i}}(\mathbb{R})(0 \leq i \leq t)$. We will use this notation throughout the paper.

Lemma 2.7 [Dekimpe et al. 2006]. Let $B, C \in M_{n}(\mathbb{R})$ be two real matrices such that $B C=C B$ and $B$ has only nonreal eigenvalues. Then the multiplicity of any real eigenvalue of $C$ must be even, which implies that $\operatorname{det}\left(I_{n}-C\right) \geq 0$.

\section{A class of infranilmanifolds with cyclic holonomy group}

This section is completely devoted to the proof of the main result of this paper:

Theorem 3.1. Let $M$ be an infranilmanifold with cyclic holonomy group $F$ generated by $x_{0}$. Let $T: F \rightarrow$ Aut $G$ be the holonomy representation and suppose -1 is not an eigenvalue of $T_{*}\left(x_{0}\right)$. Then $N(f)=|L(f)|$ for any continuous map $f: M \rightarrow M$.

The proof will be based on Theorem 2.3, and therefore we have to examine the sign of the determinants $\operatorname{det}\left(I_{n}-T_{*}\left(x_{0}^{m}\right) \mathfrak{D}_{*}\right)$ for $0 \leq m<2^{r} k$. Using the notations 
of the previous section, we have

$$
\begin{aligned}
\operatorname{det}\left(I_{n}-T_{*}\left(x_{0}^{m}\right) \mathfrak{D}_{*}\right) & =\operatorname{det}\left(I_{n}-P T_{*}\left(x_{0}^{m}\right) P^{-1} P \mathfrak{D}_{*} P^{-1}\right) \\
& =\operatorname{det}\left(I_{n_{0}}-A_{0}^{m} D_{0}\right) \cdots \operatorname{det}\left(I_{n_{t}}-A_{t}^{m} D_{t}\right) .
\end{aligned}
$$

Thus it suffices to consider the determinants $\operatorname{det}\left(I_{n_{i}}-A_{i}^{m} D_{i}\right)$ separately. This allows us to reduce our investigation to the study of the sign of determinants of the form

$$
\operatorname{det}\left(I_{n}-A^{m} D\right) \quad\left(0 \leq m<2^{s} d\right),
$$

where

(1) $2^{s} d \mid 2^{r} k$, with $d$ an odd integer,

(2) $A^{2^{s} d}=I_{n}$,

(3) each eigenvalue of $A$ is exactly of order $2^{s} d$, and

(4) $D A=A^{l} D$ for some $l$, with $0 \leq l<2^{s} d$.

We will distinguish several cases, depending on the possible values of $s, d$ and $l$. We will not need the case $s=1$ and $d=1$, which corresponds to an eigenvalue -1 in $T_{*}\left(x_{0}\right)$.

To deal with all possible cases, we have to prove a series of lemmas. In fact, we are going to use the following scheme in our treatment:

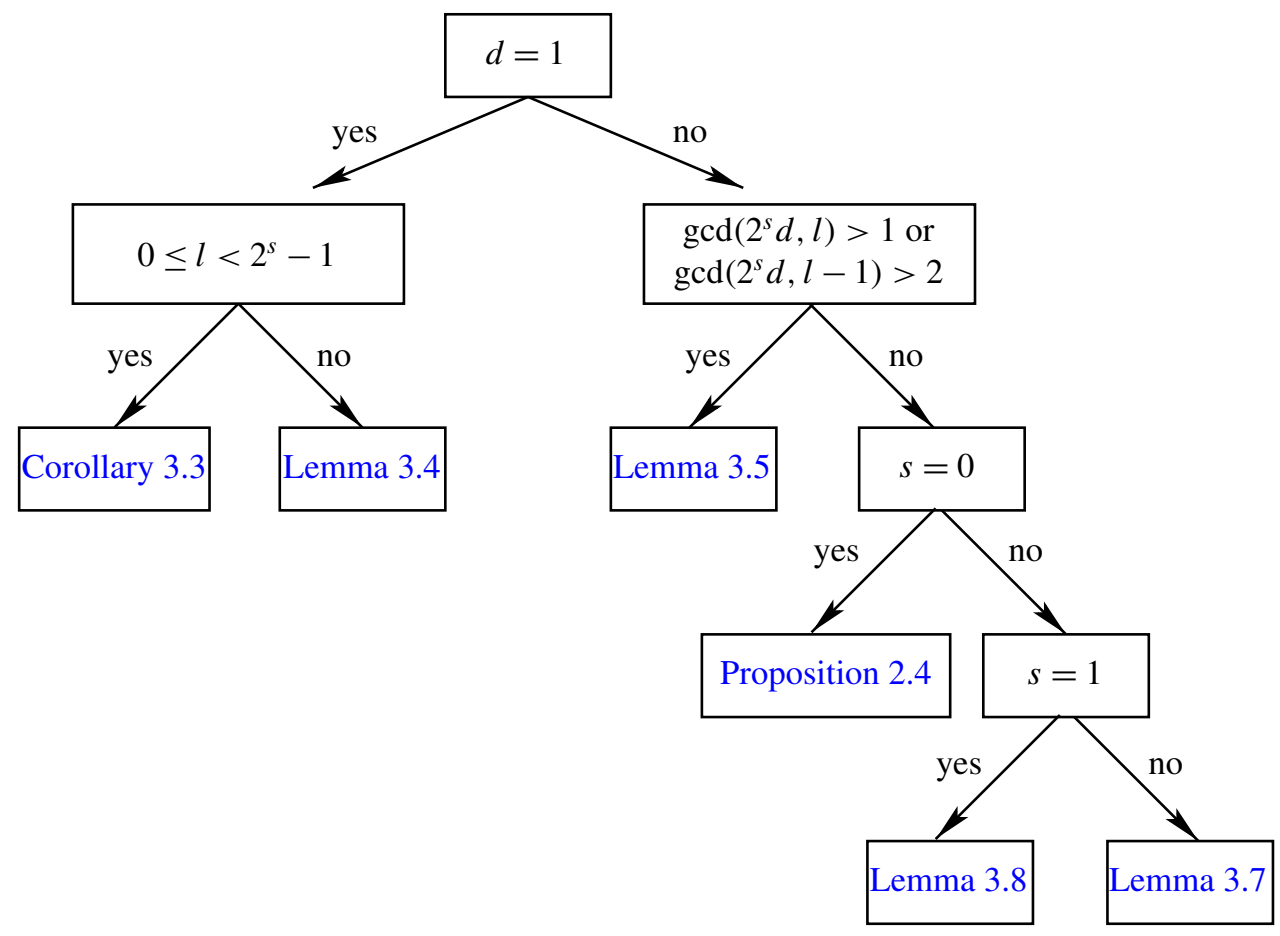


We start by looking at matrices of order $2^{s}$ (the left-hand side of the scheme) and distinguish two cases depending on the value of $l$.

Lemma 3.2. Suppose $A \in \mathrm{GL}(n, \mathbb{R})$ only has eigenvalues of order $2^{s}$ with $s \geq 2$. Suppose $D \in M_{n}(\mathbb{R})$ such that $D A=A^{l} D$ with $0 \leq l<2^{s}-1$. Then the multiplicity of any real eigenvalue of $D$ must be even.

Proof. We work by induction on $s$, starting with $s=2$, so $l$ can be equal to 0,1 or 2. If $l=0$ then $D A=D$ or $D\left(A-I_{n}\right)=0_{n}$. Since $A$ does not have 1 as an eigenvalue, $A-I_{n}$ is invertible and so $D=0_{n}$. Note that $n$ is even since $A$ only has nonreal eigenvalues and so in this case the lemma holds. If $l=1$ we can apply Lemma 2.7 to $A$ and $D$. Finally if $l=2$ we have $D A^{2}=A^{4} D=D$ and again $D=0_{n}$ since $A^{2}=-I_{n}$.

Now assume that $s>2$ and that the lemma holds for smaller values of $s$. Since $D A=A^{l} D$ we also have $D A^{2}=\left(A^{2}\right)^{l} D$ and we can apply the induction hypothesis on $A^{2}$, which is of order $2^{s-1}$. We conclude that the lemma holds for any $l$ with $0 \leq l<2^{s-1}-1$; but it already holds also for any $l$ with $2^{s-1} \leq l<2^{s}-1$. Indeed for such $l$ we can consider $l^{\prime}=l-2^{s-1}$, then $0 \leq l^{\prime}<2^{s-1}-1$ and we obtain $D A^{2}=\left(A^{2}\right)^{l} D=\left(A^{2}\right)^{l^{\prime}+2^{s-1}} D=\left(A^{2}\right)^{l^{\prime}} D$. So, we can again apply the induction hypothesis.

There is still one case left, namely $l=2^{s-1}-1$. Then

$$
D A^{2^{s-1}-1}=\left(A^{2^{s-1}-1}\right)^{\left(2^{s-1}-1\right)} D=A^{\left(2^{s-1}-1\right)^{2}} D .
$$

Now $\left(2^{s-1}-1\right)^{2}=2^{2 s-2}-2 \cdot 2^{s-1}+1=2^{s}\left(2^{s-2}-1\right)+1$ and therefore $D A^{2^{s-1}-1}=$ $A D$. This implies that $D\left(A+A^{2^{s-1}-1}\right)=\left(A+A^{2^{s-1}-1}\right) D$. If we can show that $A+A^{2^{s-1}-1}$ only has nonreal eigenvalues, we can apply Lemma 2.7 to deduce that the lemma also holds in this case. Since $A$ only has eigenvalues of order $2^{s}$ we know that $A+A^{2^{s-1}-1}$ only has eigenvalues of the form

$$
e^{2 \pi i t / 2^{s}}+e^{2 \pi i t / 2^{s}\left(2^{s-1}-1\right)}
$$

with $\operatorname{gcd}\left(t, 2^{s}\right)=1$. Therefore $t$ is odd and the imaginary part of such an eigenvalue is equal to

$$
\begin{aligned}
\sin \frac{2 \pi t}{2^{s}}+\sin \left(\frac{2 \pi t}{2^{s}}\left(2^{s-1}-1\right)\right) & =\sin \frac{2 \pi t}{2^{s}}+\sin \left(\pi t-\frac{2 \pi t}{2^{s}}\right) \\
& =\sin \frac{2 \pi t}{2^{s}}+\sin \left(\pi-\frac{2 \pi t}{2^{s}}\right)=2 \sin \frac{2 \pi t}{2^{s}} .
\end{aligned}
$$

Note that $2 \pi t / 2^{s}$ cannot be equal to $0, \pi$ or $2 \pi$ since $\operatorname{gcd}\left(t, 2^{s}\right)=1$. Therefore the eigenvalues of $A+A^{2^{s-1}-1}$ are always nonreal.

Corollary 3.3. For $A$ and $D$ as in Lemma 3.2 we have $\operatorname{det}\left(I_{n}-A^{m} D\right) \geq 0$ for any $0 \leq m<2^{s}$. 
Proof. Let $D^{\prime}=A^{m} D$ for any $0 \leq m<2^{s}$. Then $A$ and $D^{\prime}$ also satisfy the requirements of Lemma 3.2 and hence the multiplicity of any real eigenvalue of $D^{\prime}$ is even. The last assertion of Lemma 2.7 yields the result.

The second case is $l=2^{s}-1$ :

Lemma 3.4. Suppose $A \in \mathrm{GL}(n, \mathbb{R})$ only has eigenvalues of order $2^{s}$ with $s \geq 2$. Suppose $D \in M_{n}(\mathbb{R})$ is such that $D A=A^{2^{s}-1} D$. Then, for any $m$ with $0 \leq m<2^{s}$,

$$
\operatorname{det}\left(I_{n}-D\right)=\operatorname{det}\left(I_{n}-A^{m} D\right)
$$

Proof. Let $\mu_{1}, \bar{\mu}_{1}, \ldots \mu_{w}, \bar{\mu}_{w}$ be the different, nonreal eigenvalues of $A$ with multiplicity $m_{1}, \ldots m_{w}$. Since $A$ is diagonalizable, there exist $Q \in \operatorname{GL}(n, \mathbb{C})$ such that

$$
Q A Q^{-1}=\left(\begin{array}{ccccc}
\mu_{1} I_{m_{1}} & 0 & \cdots & 0 & 0 \\
0 & \bar{\mu}_{1} I_{m_{1}} & \cdots & 0 & 0 \\
\vdots & & \ddots & & \vdots \\
0 & 0 & \cdots & \mu_{w} I_{m_{w}} & 0 \\
0 & 0 & \cdots & 0 & \bar{\mu}_{w} I_{m_{w}}
\end{array}\right)
$$

Since all the eigenvalues are of order $2^{s}$ this implies that

$$
Q A^{2^{s}-1} Q^{-1}=\left(\begin{array}{ccccc}
\bar{\mu}_{1} I_{m_{1}} & 0 & \cdots & 0 & 0 \\
0 & \mu_{1} I_{m_{1}} & \cdots & 0 & 0 \\
\vdots & & \ddots & & \vdots \\
0 & 0 & \cdots & \bar{\mu}_{w} I_{m_{w}} & 0 \\
0 & 0 & \cdots & 0 & \mu_{w} I_{m_{w}}
\end{array}\right)
$$

One can easily verify that because of $D A=A^{2^{s-1}} D$ we obtain that

$$
Q D Q^{-1}=\left(\begin{array}{ccccc}
0 & D_{i_{1}} & \cdots & 0 & 0 \\
D_{i_{1}}^{\prime} & 0 & \cdots & 0 & 0 \\
\vdots & & \ddots & & \vdots \\
0 & 0 & \cdots & 0 & D_{i_{w}} \\
0 & 0 & \cdots & D_{i_{w}}^{\prime} & 0
\end{array}\right)
$$

with $D_{i_{j}}, D_{i_{j}}^{\prime} \in M_{m_{j}}(\mathbb{C}), 1 \leq j \leq w$. Then, since the eigenvalues of $A_{i}$ are roots of unity, we can calculate $\operatorname{det}\left(I_{n}-A^{m} D\right)$ for any $m$ with $0 \leq m<2^{s}$ : 


$$
\begin{aligned}
& \operatorname{det}\left(I_{n}-A^{m} D\right)=\operatorname{det}\left(I_{n}-Q A^{m} Q^{-1} Q D Q^{-1}\right) \\
& =\operatorname{det}\left(\begin{array}{ccccc}
I_{m_{1}} & -\left(\mu_{1}\right)^{m} D_{i_{1}} \cdots & 0 & 0 \\
-\left(\bar{\mu}_{1}\right)^{m} D_{i_{1}}^{\prime} & I_{m_{1}} & \cdots & 0 & 0 \\
\vdots & & \ddots & & \vdots \\
0 & 0 & \cdots & I_{m_{w}} & -\left(\mu_{w}\right)^{m} D_{i_{w}} \\
0 & 0 & \cdots & -\left(\bar{\mu}_{w}\right)^{m} D_{i_{w}}^{\prime} & I_{m_{w}}
\end{array}\right) \\
& =\mu_{1}^{m m_{1}} \cdots \mu_{w}^{m m_{w}} \operatorname{det}\left(\begin{array}{ccccc}
\bar{\mu}_{1}^{m} I_{m_{1}} & -D_{i_{1}} \cdots & 0 & 0 \\
-\left(\bar{\mu}_{1}\right)^{m} D_{i_{1}}^{\prime} & I_{m_{1}} & \cdots & 0 & 0 \\
\vdots & & \ddots & & \vdots \\
0 & 0 & \cdots & \bar{\mu}_{w}^{m} I_{m_{w}} & -D_{i_{w}} \\
0 & 0 & \cdots & -\left(\bar{\mu}_{w}\right)^{m} D_{i_{w}}^{\prime} & I_{m_{w}}
\end{array}\right) \\
& =\left(\mu_{1} \bar{\mu}_{1}\right)^{m m_{1}} \cdots\left(\mu_{w} \bar{\mu}_{w}\right)^{m m_{w}} \operatorname{det}\left(\begin{array}{ccccc}
I_{m_{1}} & -D_{i_{1}} & \cdots & 0 & 0 \\
-D_{i_{1}}^{\prime} & I_{m_{1}} & \cdots & 0 & 0 \\
\vdots & & \ddots & \vdots \\
0 & 0 & \cdots & I_{m_{w}} & D_{i_{w}} \\
0 & 0 & \cdots & D_{i_{w}}^{\prime} & I_{m_{w}}
\end{array}\right) \\
& =\operatorname{det}\left(I_{n}-D\right) \text {. }
\end{aligned}
$$

From now on we consider matrices of order $2^{s} d$, with $d>1$. Again we distinguish several cases, depending on the value of $l$. We first consider $l$ for which $\operatorname{gcd}\left(2^{s} d, l\right)>1$ or $\operatorname{gcd}\left(2^{s} d, l-1\right)>2$.

Lemma 3.5. Let $d>1$ be an odd integer and let $s \geq 0$. Suppose $A \in \operatorname{GL}(n, \mathbb{R})$ only has eigenvalues of order $2^{s} d$ and suppose $D \in M_{n}(\mathbb{R})$ is such that $D A=A^{l} D$, with $0 \leq l<2^{s} d$.

If $\operatorname{gcd}\left(2^{s} d, l\right)>1$ or $\operatorname{gcd}\left(2^{s} d, l-1\right)>2$ then $\operatorname{det}\left(I_{n}-A^{m} D\right) \geq 0$ for any $m$.

Proof. Assume first that $\operatorname{gcd}\left(2^{s} d, l\right)=l_{1}>1$ and that $l=l_{1} l_{2}$ and $2^{s} d=l_{1} d^{\prime}$. Then

$$
D A^{d^{\prime}}=\left(A^{d^{\prime}}\right)^{l} D=A^{2^{s} d l_{2}} D=D \text {. }
$$

Now 1 is not an eigenvalue of $A^{d^{\prime}}$, since $d^{\prime}<2^{s} d$ and the eigenvalues of $A$ have order $2^{s} d$. This implies as before that $D=0_{n}$ and $\operatorname{det}\left(I_{n}-A^{m} D\right)=1 \geq 0$ for any $m$.

Next assume that $\operatorname{gcd}\left(2^{s} d, l-1\right)=l_{1}>2$ and that $l-1=l_{1} l_{2}$ and $2^{s} d=l_{1} d^{\prime}$. Then

$$
D A^{d^{\prime}}=\left(A^{d^{\prime}}\right)^{l} D=A^{d^{\prime}\left(1+l_{1} l_{2}\right)} D=A^{d^{\prime}+2^{s} d l_{2}} D=A^{d^{\prime}} D
$$


Now $A^{d^{\prime}}$ only has nonreal eigenvalues, since $d^{\prime}<2^{s-1} d$ and the eigenvalues of $A$ have order $2^{s} d$. So we can apply Lemma 2.7 to finish the proof.

Finally, we must consider the situation where $\operatorname{gcd}\left(2^{s} d, l\right)=1$ and $\operatorname{gcd}\left(2^{s} d, l-1\right)$ is at most 2. If moreover $s=0$, then $\operatorname{gcd}(d, l-1)=1$ since $d$ is odd. In this case one can easily verify that any power of $A$ does not commute with $D$ and so we can apply Proposition 2.4. (See also the proof of [Dekimpe et al. 2006, Lemma 2.4].) On the other hand, if $s \geq 1$ then $\operatorname{gcd}\left(2^{s} d, l-1\right)=2$, since $\operatorname{gcd}\left(2^{s} d, l\right)=1$ implies that $l$ is odd.

The following lemma is useful to solve the second case.

Lemma 3.6. Let $d>1$ be an odd integer and let $s \geq 1$. Suppose $A \in \operatorname{GL}(n, \mathbb{R})$ only has eigenvalues of order $2^{s} d$ and suppose $D \in M_{n}(\mathbb{R})$ is such that $D A=A^{l} D$ with $0 \leq l<2^{s} d$.

If $\operatorname{gcd}\left(2^{s} d, l-1\right)=2$ then $\operatorname{det}\left(I_{n}-D\right)=\operatorname{det}\left(I_{n}-A^{2 m} D\right)$ and $\operatorname{det}\left(I_{n}-A D\right)=$ $\operatorname{det}\left(I_{n}-A^{2 m+1} D\right)$ for any $m$.

Proof. We can establish for any $m$ the following relations between the determinants.

$$
\begin{aligned}
\operatorname{det}\left(I_{n}-D\right) & =\operatorname{det}\left(A^{m}-D A^{m}\right) \operatorname{det}\left(A^{-m}\right) \\
& =\operatorname{det}\left(A^{-m}\right) \operatorname{det}\left(A^{m}-A^{m l} D\right)=\operatorname{det}\left(I_{n}-A^{m(l-1)} D\right) .
\end{aligned}
$$

Since $\operatorname{gcd}\left(l-1,2^{s} d\right)=2$, the group generated by $A^{l-1}$ is of order $2^{s-1} d$ and thus consists of all even powers of $A$. Hence $\operatorname{det}\left(I_{n}-D\right)=\operatorname{det}\left(I_{n}-A^{2 m} D\right)$ for any $m$.

The second part can be proved analogously with $D^{\prime}=A D$.

With this last lemma, we can prove the following lemma for $s=1$.

Lemma 3.7. Let $d>1$ be an odd integer. Suppose $A \in \mathrm{GL}(n, \mathbb{R})$ only has eigenvalues of order $2 d$ and suppose $D \in M_{n}(\mathbb{R})$ is such that $D A=A^{l} D$ with $0 \leq l<2 d$.

If $\operatorname{gcd}(2 d, l)=1$ and $\operatorname{gcd}(2 d, l-1)=2$, then $\operatorname{det}\left(I_{n}-A^{m} D_{i}\right) \geq 0$ for any $m$ or $\operatorname{det}\left(I_{n}-A^{m} D_{i}\right) \leq 0$ for any $m$.

Proof. Because of Lemma 3.6 we only have to prove the lemma for $\operatorname{det}\left(I_{n}-D\right)$ and $\operatorname{det}\left(I_{n}-A D\right)$. Moreover since $d$ is odd and the eigenvalues of $A^{d}$ are of order 2, it suffices to prove the lemma for $\operatorname{det}\left(I_{n}-D\right)$ and $\operatorname{det}\left(I_{n}-A D\right)=\operatorname{det}\left(I_{n}-A^{d} D\right)=$ $\operatorname{det}\left(I_{n}+D\right)$.

Since $\operatorname{gcd}(2 d, l)=1$, Euler's theorem tells us that $l^{\phi(2 d)} \equiv 1 \bmod 2 d$. Hence

$$
D^{\phi(2 d)} A=D^{\phi(2 d)-1} A^{l} D=D^{\phi(2 d)-2} A^{l^{2}} D^{2}=\cdots=A^{l^{\phi(2 d)}} D^{\phi(2 d)}=A D^{\phi(2 d)} .
$$

So we obtain that, because of Lemma 2.7, the multiplicity of the real eigenvalues of $D^{\phi(2 d)}$ must be even.

Suppose that $\lambda_{1}, \ldots, \lambda_{v}$ are the real, positive eigenvalues of $D^{\phi(2 d)}$ each with even multiplicity $m_{1}, \ldots, m_{v}$; that $\gamma_{1}, \ldots, \gamma_{t_{1}}$ are the real, strictly negative eigenvalues of $D^{\phi(2 d)}$; and that $\mu_{1}, \bar{\mu}_{1}, \ldots, \mu_{t_{2}}, \bar{\mu}_{t_{2}}$ are the nonreal eigenvalues of $D^{\phi(2 d)}$. 
The eigenvalues of $D$ must be $\phi(2 d)$-th roots of these eigenvalues of $D^{\phi(2 d)}$. Note that $\phi(2 d)$ is an even integer since $d>1$, and therefore a $\phi(2 d)$-th root of $\gamma_{i}$ or $\mu_{k}$ is always nonreal, Whereas the $\phi(2 d)$-th root of the $\lambda_{j}$ can be real or not, say $\alpha_{j}$. But if $\alpha_{j}$ is an eigenvalue of $D$, then $\bar{\alpha}_{j}$ also has to be an eigenvalue of $D$. Now

$$
\left(\bar{\alpha}_{j}\right)^{\phi(2 d)}=\overline{\alpha_{j}^{\phi(2 d)}}=\lambda_{j},
$$

so $\bar{\alpha}_{j}$ has to be a $\phi(2 d)$-th root of the same $\lambda_{j}$. Since the $m_{j}$ are even, this implies that the number of real eigenvalues of $D$ coming from $\lambda_{j}$, for $1 \leq j \leq v$, is even.

For each $j, 1 \leq j \leq v$, denote the positive real $\phi(2 d)$-th root by $\delta_{j}$ (and the negative real root by $-\delta_{j}$ ). We denote the multiplicity of $\delta_{j}$ as an eigenvalue of $D$ by $r_{j}$, and that of $-\delta_{j}$ by $s_{j}$. It is of course possible that $\delta_{j}$ or $-\delta_{j}$ is not an eigenvalue of $D$. In this case we take its multiplicity to be 0 . We then always have $r_{j}+s_{j} \in 2 \mathbb{Z}$.

Using the arguments in the proof of Corollary 3.3 and the information above, we know that the only factors that matter are

$$
\left(1-\delta_{1}\right)^{r_{1}} \cdots\left(1-\delta_{v}\right)^{r_{v}}\left(1+\delta_{1}\right)^{s_{1}} \cdots\left(1+\delta_{v}\right)^{s_{v}}
$$

in $\operatorname{det}\left(I_{n}-D\right)$ and

$$
\left(1+\delta_{1}\right)^{r_{1}} \cdots\left(1+\delta_{v}\right)^{r_{v}}\left(1-\delta_{1}\right)^{s_{1}} \cdots\left(1-\delta_{v}\right)^{s_{v}}
$$

in $\operatorname{det}\left(I_{n}+D\right)$.

For each $i \in\{1, \ldots, v\}$ there is in $\operatorname{det}\left(I_{n}-D\right)$ a factor of the form $\left(1-\delta_{i}\right)^{r_{i}}(1+$ $\left.\delta_{i}\right)^{s_{i}}$ and in $\operatorname{det}\left(I_{n}+D\right)$ there is a factor of the form $\left(1+\delta_{i}\right)^{r_{i}}\left(1-\delta_{i}\right)^{s_{i}}$. Suppose that $r_{i} \geq s_{i}$ (the other case is completely similar). Then

$$
\left(1-\delta_{i}\right)^{r_{i}}\left(1+\delta_{i}\right)^{s_{i}}=\left(1-\delta_{i}\right)^{\left(r_{i}-s_{i}\right)}\left(1-\delta_{i}\right)^{s_{i}}\left(1+\delta_{i}\right)^{s_{i}}=\left(1-\delta_{i}\right)^{r_{i}-s_{i}}\left(1-\delta_{i}^{2}\right)^{s_{i}}
$$

and

$$
\left(1+\delta_{i}\right)^{r_{i}}\left(1-\delta_{i}\right)^{s_{i}}=\left(1+\delta_{i}\right)^{r_{i}-s_{i}}\left(1-\delta_{i}^{2}\right)^{s_{i}} .
$$

Since $r_{i}+s_{i}$ is an even integer, so is $r_{i}-s_{i}$. So in both cases the first factor is positive and the second factor is the same. This ends the proof of this lemma.

Finally we can prove the following lemma for $s \geq 2$.

Lemma 3.8. Let $d>1$ be an odd integer and $s \geq 2$. Suppose $A \in \operatorname{GL}(n, \mathbb{R})$ only has eigenvalues of order $2^{s} d$ and suppose $D \in M_{n}(\mathbb{R})$ such that $D A=A^{l} D$ with $0 \leq l<2^{s} d$.

- If $\operatorname{gcd}\left(2^{s} d, l-1\right)=2$ and $l \not \equiv 2^{s}-1 \bmod 2^{s}$, then $\operatorname{det}\left(I_{n}-A^{m} D\right) \geq 0$ for any $m$.

- If $\operatorname{gcd}\left(2^{s} d, l-1\right)=2$ and $l \equiv 2^{s}-1 \bmod 2^{s}$, then $\operatorname{det}\left(I_{n}-D\right)=\operatorname{det}\left(I_{n}-A^{m} D\right)$ for any $m$. 
Proof. Because of Lemma 3.6 we only have to prove the lemma for $\operatorname{det}\left(I_{n}-D\right)$ and $\operatorname{det}\left(I_{n}-A D\right)$. The same lemma also implies that $\operatorname{det}\left(I_{n}-A D\right)=\operatorname{det}\left(I_{n}-A^{d} D\right)$, since $d$ is odd. Now $A^{d}$ only has eigenvalues of order $2^{s}$ and if $l \not \equiv 2^{s}-1 \bmod 2^{s}$ we can apply Corollary 3.3 to $D$ and $A^{d}$. If $l \equiv 2^{s}-1 \bmod 2^{s}$, on the other hand, we can apply Lemma 3.4 to $D$ and $A^{d}$.

Proof of Theorem 3.1. Denote the order of $F$ by $2^{r} k$ with $k$ an odd integer. Let $(\delta, \mathfrak{D})$ be a homotopy lift of $f$ and suppose that $\mathfrak{D}_{*} T_{*}\left(x_{0}\right)=T_{*}\left(x_{0}^{l}\right) \mathfrak{D}_{*}$. Suppose $d_{0}, d_{1}, \ldots, d_{t}$ are the divisors of $2^{r} k$ and suppose $1=d_{0}<d_{1}<\cdots<d_{t}=2^{r} k$. Because of Lemma 2.5 and of the condition on $T_{*}\left(x_{0}\right)$, there exists $n_{0}, n_{2}, \ldots, n_{t} \in$ $\mathbb{N}$ and $P \in \mathrm{GL}(n, \mathbb{R})$ such that $n_{0}+n_{2}+\cdots+n_{t}=n$ and

$$
P T_{*}\left(x_{0}\right) P^{-1}=\left(\begin{array}{cccc}
A_{0} & 0 & \cdots & 0 \\
0 & A_{2} & \cdots & 0 \\
\vdots & \vdots & \ddots & \vdots \\
0 & 0 & \cdots & A_{t}
\end{array}\right),
$$

with $A_{i} \in \mathrm{GL}\left(n_{i}, \mathbb{R}\right)$ having only eigenvalues of order $d_{i}$, for $0 \leq i \leq t$. Note that $n_{1}=0$ since -1 is not an eigenvalue of $T_{*}\left(x_{0}\right)$. Because of Lemma 2.6 we also have

$$
P \mathfrak{D}_{*} P^{-1}=\left(\begin{array}{cccc}
D_{0} & 0 & \cdots & 0 \\
* & D_{2} & \cdots & 0 \\
\vdots & \vdots & \ddots & \vdots \\
* & * & \cdots & D_{t}
\end{array}\right)
$$

with $D_{i} \in M_{n_{i}}(\mathbb{R})$ for $0 \leq i \leq t$, and $*$ can be any block of real numbers.

If we want to use Theorem 2.3, we have to calculate

$$
\operatorname{det}\left(I_{n}-T_{*}\left(x_{0}^{m}\right) \mathfrak{D}_{*}\right)=\operatorname{det}\left(I_{n_{0}}-A_{0}^{m} D_{0}\right) \cdots \operatorname{det}\left(I_{n_{t}}-A_{t}^{m} D_{t}\right)
$$

for all $m$ such that $0 \leq m<2^{r} k$. As explained before, we consider all the factors above separately (so we fix an $i$ and see what happens when $m$ varies). If $d_{i}=2^{s}$, with $s \geq 2$, then we can, depending on $l$, apply Corollary 3.3 or Lemma 3.4 to show that all these factors have the same sign. In case $s$ is zero $(i=0)$, the value of $\operatorname{det}\left(I_{n_{0}}-A_{0}^{m} D_{0}\right)$ does not depend on $m$. By our assumption, the case $s=1$ does not occur.

If $d_{i}$ is not a power of 2 then we can, again depending on the value of $l$ (see the scheme on page 143 and the discussion immediately before Lemma 3.6), apply Lemma 3.5, Proposition 2.4, Lemma 3.7 or Lemma 3.8 to show that these factors also have the same sign.

So in each case we conclude that the condition in Theorem 2.3 is satisfied and for each $f$ we have $N(f)=|L(f)|$. 
Remark 3.9. (a) The condition that -1 is not an eigenvalue of $T_{*}\left(x_{0}\right)$ is crucial given Anosov's counterexample on the Klein Bottle, which has $\mathbb{Z}_{2}$ as its holonomy group. In the following section we go deeper into this.

(b) In [Dekimpe et al. 2006] we proved an analogue of Theorem 3.1: namely, that Anosov's theorem holds for infranilmanifolds with abelian holonomy group of odd order. In this case -1 is never an eigenvalue.

(c) In [Dekimpe et al. 2004] we proved that Anosov's theorem holds for orientable generalized Hantzsche-Wendt manifolds. This implies that it is not straightforward to generalize Theorem 3.1 to infranilmanifolds with other holonomy groups if -1 is an eigenvalue.

In the following sections we examine the infranilmanifolds $M$ with cyclic holonomy group, for which the holonomy representation does not satisfy the condition of Theorem 3.1. We have to distinguish two cases for these manifolds. If $M$ is a flat manifold, we are always able to construct a continuous map $f: M \rightarrow M$ such that $N(f) \neq|L(f)|$. So in the case of flat manifolds with cyclic holonomy group, we have a complete picture. On the other hand we also show in Section 5 that in general this construction of counterexamples is not possible for arbitrary infranilmanifolds with cyclic holonomy group, since we have found a nonorientable infranilmanifold $M_{1}$ for which Anosov's theorem holds, but nevertheless the holonomy representation of $M_{1}$ does not satisfy the condition of Theorem 3.1.

\section{The main result is sharp for flat manifolds}

In order to construct a continuous map $f$ on a flat manifold with cyclic holonomy group which does not satisfy the condition in Theorem 3.1, we will also distinguish two cases, based on the orientability of the manifold. Let us therefore first recall the following.

Remark 4.1. Suppose $M$ is an infranilmanifold with holonomy group $F$ and associated holonomy representation $T: F \rightarrow$ Aut $G$. Then $M$ is an orientable manifold if and only if $\operatorname{det} T_{*}(x)=1$ for all $x \in F$. If there exists an element $x \in F$ such that $\operatorname{det} T_{*}(x)=-1, M$ is nonorientable. For more background see [Brown 1982, page 211; Dekimpe 1996, page 135].

Thus the manifolds that satisfy the conditions of Theorem 3.1 are orientable. We first consider the nonorientable case and prove the following proposition, which in fact holds in the case of general (not necessarily cyclic) holonomy groups.

Proposition 4.2. Let $M$ be a n-dimensional nonorientable flat manifold. Then there always exists a continuous map $f: M \rightarrow M$ such that $N(f) \neq|L(f)|$.

Proof. Suppose that $F$ is the holonomy group of $M$ and $T: F \rightarrow$ Aut $\mathbb{R}^{n}$ is the associated holonomy representation. Since $M$ is a flat manifold we can realize 
$\pi_{1}(M)$ as a discrete subgroup of Aff $\mathbb{R}^{n}$ in such a way that its subgroup of translations is a uniform lattice in the full group of translations $\mathbb{R}^{n}$ and forms a subgroup of finite index in the whole group $\pi_{1}(M)$. Therefore we may assume that $\pi_{1}(M)$ is generated by the elements $\left(e_{1}, I_{n}\right), \ldots,\left(e_{n}, I_{n}\right),\left(a_{1}, A_{1}\right), \ldots,\left(a_{k}, A_{k}\right)$, where $e_{i}$ has 1 on the $i$-th place and 0 everywhere else. Recall that $e_{i}, a_{j}$ are the translational parts and $I_{n}, A_{j} \in \mathrm{GL}(n, \mathbb{Z})$ are the linear parts. Without loss of generality, we can assume that all $a_{i}$ are rational numbers (in fact a detailed explanation for this is given in the proof of the next proposition for cyclic groups, but the argument there generalizes to the general case). Let $k$ be a positive integer which is a common multiple of all denominators of the translational parts $a_{j}$.

To prove the proposition we verify that $\left(0,(k+1) I_{n}\right)$ induces a continuous map $f$ on $M$ and that $N(f) \neq|L(f)|$. Because of Theorem 2.1, we know that $\left(0,(k+1) I_{n}\right)$ induces a continuous map $f$ if

$$
\left(0,(k+1) I_{n}\right) \pi_{1}(M)\left(0,(k+1) I_{n}\right)^{-1}=\left(0,(k+1) I_{n}\right) \pi_{1}(M)\left(0, \frac{1}{k+1} I_{n}\right) \subseteq \pi_{1}(M) .
$$

Now for any $j, 1 \leq j \leq k$ we have

$$
\begin{aligned}
\left(0,(k+1) I_{n}\right)\left(a_{j}, A_{j}\right)\left(0, \frac{1}{k+1} I_{n}\right) & =\left((k+1) a_{j},(k+1) A_{j}\right)\left(0, \frac{1}{k+1} I_{n}\right) \\
& =\left((k+1) a_{j}, A_{j}\right) \\
& =\left(k a_{j}, I_{n}\right)\left(a_{j}, A_{j}\right) .
\end{aligned}
$$

Because of the choice of $k$, this element too belongs to $\pi_{1}(M)$. One can easily verify that the same is true for any $\left(e_{i}, I_{n}\right)$ and so we may conclude that $\left(0,(k+1) I_{n}\right)$ induces a continuous map $f$ on $M$.

To show that the proposition holds for this map $f$, we will use Theorem 2.3. Recall that since we work with flat manifolds we have

$$
\left((k+1) I_{n}\right)_{*}=(k+1) I_{n},
$$

so

$$
\operatorname{det}\left(I_{n}-\left((k+1) I_{n}\right)_{*}\right)=(-k)^{n} .
$$

Since $M$ is nonorientable we know that there exists an $x \in F$ such that $\operatorname{det} T_{*}(x)=$ -1 . For this $x$ we can construct $P \in \mathrm{GL}(n, \mathbb{Q})$ such that

$$
P T_{*}(x) P^{-1}=\left(\begin{array}{rrr}
I_{s} & 0 & 0 \\
0 & -I_{t} & 0 \\
0 & 0 & C
\end{array}\right),
$$

where $s \geq 0, t$ is an odd integer and $C$ is an $(n-(s+t)) \times(n-(s+t))$ rational matrix having only nonreal eigenvalues. This implies that 


$$
\begin{aligned}
\operatorname{det}\left(I_{n}-T_{*}(x)\left((k+1) I_{n}\right)_{*}\right) & =\operatorname{det}\left(I_{n}-P T_{*}(x) P^{-1} P(k+1) I_{n} P^{-1}\right) \\
& =\operatorname{det}\left(\left(\begin{array}{rrr}
I_{s} & 0 & 0 \\
0 & I_{t} & 0 \\
0 & 0 & I_{n-s-t}
\end{array}\right)-\left(\begin{array}{rrr}
I_{s} & 0 & 0 \\
0 & -I_{t} & 0 \\
0 & 0 & C
\end{array}\right)(k+1)\right) \\
& =(-k)^{s}(k+2)^{t} \operatorname{det}\left(I_{n-s-t}-(k+1) C\right) .
\end{aligned}
$$

As before, the third factor is always strict positive since $(k+1) C$ only has nonreal eigenvalues. Note also that, by the construction of $C, n-s-t$ must be even. So the sign of

$$
\operatorname{det}\left(I_{n}-\left((k+1) I_{n}\right)_{*}\right)=(-k)^{s}(-k)^{t}(-k)^{n-s-t}
$$

is completely determined by the factor $(-k)^{s}(-k)^{t}$. Since $t$ is odd this implies that $\operatorname{det}\left(I_{n}-\left((k+1) I_{n}\right)_{*}\right)$ and $\operatorname{det}\left(I_{n}-T_{*}(x)\left((k+1) I_{n}\right)_{*}\right)$ have a different sign and so Theorem 2.3 implies that for this map $N(f) \neq|L(f)|$.

Remark 4.3. (a) This result generalizes [Dekimpe et al. 2004, Theorem 4.1].

(b) In the proof we realized $\pi_{1}(M)$ as a subgroup of Aff $\mathbb{R}^{n}$ rather than as a subgroup of the group of isometries Isom $\mathbb{R}^{n}$ - equivalently, we realized $M$ as a complete affinely flat manifold, rather than as a (diffeomorphic) flat Riemannian manifold - but this does not play a role in our discussion. The same holds for the next proposition.

(c) Proposition 4.2 only holds for flat manifolds since in the next section we will construct an infranilmanifold $M_{1}$ which is nonorientable but nevertheless Anosov's theorem still holds for it.

For the case of flat orientable manifolds we will restrict ourselves again to flat manifolds with cyclic holonomy group and not satisfying the condition in Theorem 3.1.

Proposition 4.4. Let $M$ be a $n$-dimensional, orientable, flat manifold with cyclic holonomy group $F$ generated by $x_{0}$. Let $T: F \rightarrow$ Aut $\mathbb{R}^{n}$ be the associated holonomy representation and suppose -1 is an eigenvalue of $T_{*}\left(x_{0}\right)$. Then there always exists a continuous map $f: M \rightarrow M$ such that $N(f) \neq|L(f)|$.

Proof. As -1 is an eigenvalue of the holonomy representation, $F$ has to be a cyclic group of even order, say $2 m$.

Also, $\pi_{1}(M)$ is an $n$-dimensional Bieberbach group with translation subgroup $Z \cong \mathbb{Z}^{n}$ and holonomy group $\mathbb{Z}_{2 m}=\left\langle x_{0}\right\rangle$, for some $m \geq 1$. The group $\pi_{1}(M)$ fits in a short exact sequence

$$
1 \rightarrow Z \cong \mathbb{Z}^{n} \rightarrow \pi_{1}(M) \rightarrow \mathbb{Z}_{2 m}=\left\langle x_{0}\right\rangle \rightarrow 1,
$$


which determines a faithful representation

$$
\varphi: \mathbb{Z}_{2 m} \rightarrow \text { Aut } Z
$$

(When viewed as a real representation, this is actually the same as the holonomy representation $T$.) With respect to a good choice of generators of the free abelian group $Z, \varphi$ is represented by block-diagonal matrices, with

$$
\varphi\left(x_{0}\right)=\left(\begin{array}{cc}
A\left(x_{0}\right) & 0 \\
* & C\left(x_{0}\right)
\end{array}\right),
$$

where $A\left(x_{0}\right)$ only has eigenvalues \pm 1 and $C\left(x_{0}\right)$ has no real eigenvalues. It follows that $A\left(x_{0}\right)$ is a matrix of order 2 and by changing our set of generators for $Z$ again if necessary, we can assume that

$$
\varphi\left(x_{0}\right)=\left(\begin{array}{ccc}
-I_{s} & 0 & 0 \\
* & I_{t} & 0 \\
* & * & C\left(x_{0}\right)
\end{array}\right),
$$

for some integers $s, t \geq 0$.

Now, -1 is an eigenvalue of $\varphi\left(x_{0}\right)$ (since $s \neq 0$ ) and we also assume that $M$ is orientable, which means that $s$ is even, thus at least 2 . Therefore, we will write

$$
\varphi\left(x_{0}\right)=\left(\begin{array}{ccccc}
-1 & 0 & 0 & 0 & 0 \\
0 & -1 & 0 & 0 & 0 \\
0 & 0 & -I_{s-2} & 0 & 0 \\
* & * & * & I_{t} & 0 \\
* & * & * & * & C\left(x_{0}\right)
\end{array}\right)
$$

The group $\pi_{1}(M)$ is determined by a 2-cocycle $f: \mathbb{Z}_{2 m} \times \mathbb{Z}_{2 m} \rightarrow \mathbb{Z}^{n}$. This means that the group $\pi_{1}(M)$ coincides with $\mathbb{Z}^{n} \times \mathbb{Z}_{2 m}$ as a set, and the product in $\pi_{1}(M)$ is given by

$$
(z, x)\left(z^{\prime}, y\right)=\left(z+\varphi(x) z^{\prime}+f(x, y), x y\right)
$$

for all $z, z^{\prime} \in \mathbb{Z}^{n}$ and $x, y \in \mathbb{Z}_{2 m}$. Any element of $H^{2}\left(\mathbb{Z}_{2 m}, \mathbb{Z}^{n}\right)$ has an order dividing $2 m$. Therefore, there exists a map $g: \mathbb{Z}_{2 m} \rightarrow \mathbb{Z}^{n}$ with $\delta g=2 m f$; that is to say,

$$
\delta g(x, y)=\varphi(x) g(y)-g(x y)+g(x)=2 m f(x, y) .
$$

It is now easy to check that

$$
\psi_{1}: \pi_{1}(M)=\mathbb{Z}^{n} \times \mathbb{Z}_{2 m} \rightarrow \text { Aff } \mathbb{R}^{n}:(z, x) \mapsto\left(z+\frac{g(x)}{2 m}, \varphi(x)\right)
$$


realizes the group $\pi_{1}(M)$ as an affine group, with its translation subgroup $Z$ mapped isomorphically onto $\mathbb{Z}^{n}$. The image of $\left(0, x_{0}\right)$ is of the form

$$
\left.\psi_{1}\left(0, x_{0}\right)=\left(\begin{array}{c}
x /(2 m) \\
y /(2 m) \\
u_{1} /(2 m) \\
u_{2} /(2 m) \\
u_{3} /(2 m)
\end{array}\right),\left(\begin{array}{ccccc}
-1 & 0 & 0 & 0 & 0 \\
0 & -1 & 0 & 0 & 0 \\
0 & 0 & -I_{s-2} & 0 & 0 \\
* & * & * & I_{t} & 0 \\
* & * & * & * & C\left(x_{0}\right)
\end{array}\right)\right)
$$

for some $x, y \in \mathbb{Z}, u_{1} \in \mathbb{Z}^{s-2}, u_{2} \in \mathbb{Z}^{t}, u_{3} \in \mathbb{Z}^{n-s-t}$.

Let $v=(-x /(4 m),-y /(4 m), 0,0,0)^{t} \in \mathbb{R}^{n}$ and take $\psi_{2}=\left(v, I_{n}\right) \psi_{1}\left(v, I_{n}\right)^{-1}$. Then, $\psi_{2}(z, 1)=\psi_{1}(z, 1)$ for all $z \in \mathbb{Z}^{n}$ and

$$
\psi_{2}\left(0, x_{0}\right)=\left(\left(\begin{array}{c}
0 \\
0 \\
u_{1} /(2 m) \\
u_{2} /(2 m) \\
u_{3} /(2 m)
\end{array}\right),\left(\begin{array}{ccccc}
-1 & 0 & 0 & 0 & 0 \\
0 & -1 & 0 & 0 & 0 \\
0 & 0 & -I_{s-2} & 0 & 0 \\
* & * & * & I_{t} & 0 \\
* & * & * & * & C\left(x_{0}\right)
\end{array}\right)\right) .
$$

There exists a rational matrix

$$
P=\left(\begin{array}{ccccc}
1 & 0 & 0 & 0 & 0 \\
0 & 1 & 0 & 0 & 0 \\
0 & 0 & I_{s} & 0 & 0 \\
* & * & * & I_{t} & 0 \\
* & * & * & 0 & I_{n-s-t}
\end{array}\right) \in \mathrm{GL}(n, \mathbb{Q})
$$

such that

$$
P \varphi\left(x_{0}\right) P^{-1}=\left(\begin{array}{ccccc}
-1 & 0 & 0 & 0 & 0 \\
0 & -1 & 0 & 0 & 0 \\
0 & 0 & -I_{s-2} & 0 & 0 \\
0 & 0 & 0 & I_{t} & 0 \\
0 & 0 & 0 & * & C\left(x_{0}\right)
\end{array}\right)
$$

Let $D_{1}$ be the matrix

$$
D_{1}=\left(\begin{array}{ccccc}
2 & 1 & 0 & 0 & 0 \\
1 & 1 & 0 & 0 & 0 \\
0 & 0 & I_{s-2} & 0 & 0 \\
0 & 0 & 0 & I_{t} & 0 \\
0 & 0 & 0 & 0 & I_{n-s-t}
\end{array}\right)
$$


It is obvious that $D_{1}$ commutes with $P \varphi\left(x_{0}\right) P^{-1}$ and thus $D_{2}=P^{-1} D_{1} P$ commutes with $\varphi\left(x_{0}\right)$. The matrix $D_{2}$ is an element of $\operatorname{GL}(n, \mathbb{Q})$ whose characteristic polynomial (which is the same as the characteristic polynomial of $D_{1}$ ) has integer coefficients and unit constant term. This implies, by a result of Porteous [1972], that there exists a positive integer $k$ such that $D_{3}=D_{2}^{k} \in \operatorname{GL}(n, \mathbb{Z})$. Now, $D_{3}$ has almost all eigenvalues equal to 1 , except two positive real eigenvalues, say $\lambda_{1}>1$ and $\lambda_{2}=\lambda_{1}{ }^{-1}<1$. Again by taking a suitable power of $D_{3}$, we obtain a new matrix $D_{4}=D_{3}^{l}$ whose two eigenvalues different from 1 are $\lambda_{1}^{l}, \lambda_{1}^{-l}$ and satisfy

$$
\lambda_{1}^{l}>2 m+1 \Rightarrow \frac{1}{\lambda_{1}^{l}}<\frac{1}{2 m+1} .
$$

Finally, let $D=(2 m+1) D_{4}$. It is obvious that $D$ still commutes with $\varphi\left(x_{0}\right)$. The matrix $D$ is of the form

$$
D=\left(\begin{array}{ccccc}
a & b & 0 & 0 & 0 \\
b & c & 0 & 0 & 0 \\
0 & 0 & (2 m+1) I_{s-2} & 0 & 0 \\
* & * & * & (2 m+1) I_{t} & 0 \\
* & * & * & 0 & (2 m+1) I_{n-s-t}
\end{array}\right),
$$

where each $*$ indicates a block with entries in $(2 m+1) \mathbb{Z}$ and the block $\left(\begin{array}{ll}a & c \\ b & d\end{array}\right)$ has two positive real eigenvalues $\mu_{1}$ and $\mu_{2}$ satisfying $\mu_{1}>(2 m+1)^{2}$ and $\mu_{2}<1$.

Conjugating with $(0, D)$ inside $\operatorname{Aff} \mathbb{R}^{n}$ induces an endomorphism of $\psi_{2}(E)$. This can be seen as follows:

(a) If $\left(z, I_{n}\right) \in \psi_{2}(E)$ (so $\left.z \in \mathbb{Z}^{n}\right)$, then $(0, D)\left(z, I_{n}\right)\left(0, D^{-1}\right)=\left(D z, I_{n}\right) \in \psi_{2}(E)$.

(b) Set

$$
t\left(x_{0}\right)=\left(0,0, \frac{u_{1}}{2}, \frac{u_{2}}{2}, \frac{u_{3}}{2}\right)^{t} .
$$

We compute the image of $\psi_{2}\left(0, x_{0}\right)=\left(t\left(x_{0}\right), \varphi\left(x_{0}\right)\right)$ :

$$
\begin{aligned}
(0, D) \psi_{2}\left(0, x_{0}\right) & =(0, D)\left(t\left(x_{0}\right), \varphi\left(x_{0}\right)\right)=\left(D t\left(x_{0}\right), D \varphi\left(x_{0}\right)\right) \\
& =\left(\left(D-I_{n}\right) t\left(x_{0}\right), I_{n}\right)\left(t\left(x_{0}\right), \varphi\left(x_{0}\right)\right)(0, D) .
\end{aligned}
$$

By the construction of $D$ and the fact that the first two entries of $t\left(x_{0}\right)$ are zero, we have $\left(D-I_{n}\right) t\left(x_{0}\right) \in \mathbb{Z}^{n}$. This implies that $(0, D)\left(t\left(x_{0}\right), \varphi\left(x_{0}\right)\right)(0, D)^{-1}$ lies in $\psi_{2}(E)$.

Let $f$ be the map on $M$ induced by conjugation with $(0, D)$. On the one hand,

$$
\operatorname{det}\left(I_{n}-D\right)=\underbrace{\left(1-\mu_{1}\right)}_{<0} \underbrace{\left(1-\mu_{2}\right)}_{>0} \underbrace{(-2 m)^{s-2}}_{>0}(-2 m)^{t} \underbrace{(-2 m)^{n-s-t}}_{>0},
$$


while, on the other,

$$
\begin{aligned}
& \operatorname{det}\left(I_{n}-\varphi\left(x_{0}\right) D\right) \\
&=\underbrace{\left(1+\mu_{1}\right)}_{>0} \underbrace{\left(1+\mu_{2}\right)}_{>0} \underbrace{(2+2 m)^{s-2}}_{>0}(-2 m)^{t} \underbrace{\operatorname{det}\left(I_{n-s-t}-(2 m+1) C\left(x_{0}\right)\right)}_{>0} .
\end{aligned}
$$

Clearly these two determinants differ in sign and Theorem 2.3 again implies that $N(f) \neq|L(f)|$.

Proposition 4.4 generalizes [Lee 1995, Example 2.6].

\section{Infranilmanifolds are more complicated}

In this section we construct an nonorientable infranilmanifold $M_{1}$ with cyclic holonomy group, whose holonomy representation does not satisfy the condition of Theorem 3.1. Nevertheless Anosov's theorem holds for $M_{1}$, which shows that the results obtained in the previous section are not true in general for infranilmanifolds.

Let $\mathfrak{g}$ be the 7-dimensional Lie algebra with basis $X_{1}, X_{2}, \ldots, X_{7}$, where the nonzero Lie brackets between basis vectors are given by

$$
\begin{gathered}
{\left[X_{1}, X_{2}\right]=X_{3}, \quad\left[X_{1}, X_{3}\right]=X_{4}, \quad\left[X_{1}, X_{4}\right]=X_{5}, \quad\left[X_{1}, X_{5}\right]=X_{6},} \\
{\left[X_{2}, X_{3}\right]=-X_{7}, \quad\left[X_{2}, X_{7}\right]=-X_{5}-X_{6}, \quad\left[X_{3}, X_{7}\right]=-X_{6} .}
\end{gathered}
$$

There is a faithful matrix representation $\rho: \mathfrak{g} \rightarrow M_{8}\left(\mathbb{R}^{n}\right)$ of this Lie algebra, given by

$$
x_{1} X_{1}+x_{2} X_{2}+\cdots+x_{7} X_{7} \mapsto\left(\begin{array}{cccccccc}
0 & x_{1} & -\frac{2}{3}\left(x_{2}+x_{3}\right) & 0 & \frac{1}{3} x_{7} & \frac{1}{3} x_{7} & 0 & x_{6} \\
0 & 0 & -\frac{2}{3} x_{2} & x_{1} & 0 & \frac{1}{3} x_{7} & 0 & x_{5} \\
0 & 0 & 0 & 0 & -\frac{1}{2} x_{2} & \frac{1}{2} x_{3} & 0 & x_{7} \\
0 & 0 & 0 & 0 & x_{1} & 0 & 0 & x_{4} \\
0 & 0 & 0 & 0 & 0 & x_{1} & 0 & x_{3} \\
0 & 0 & 0 & 0 & 0 & 0 & 0 & x_{2} \\
0 & 0 & 0 & 0 & 0 & 0 & 0 & x_{1} \\
0 & 0 & 0 & 0 & 0 & 0 & 0 & 0
\end{array}\right) \text {. }
$$

The reader might use this representation in checking the claims that follow.

Let exp : $\mathfrak{g} \rightarrow G$ denote the exponential map from the nilpotent Lie algebra $\mathfrak{g}$ to the corresponding simply connected, connected nilpotent Lie group $G$. (Note that $\rho$ lifts to a matrix representation of $G$ ). Consider

$$
\begin{gathered}
a_{1}=\exp \left(X_{1}\right), \quad a_{2}=\exp \left(X_{2}\right), \quad a_{3}=\exp \left(\frac{1}{2} X_{3}\right), \quad a_{4}=\exp \left(\frac{1}{8} X_{4}\right), \\
a_{5}=\exp \left(\frac{1}{48} X_{5}\right), \quad a_{6}=\exp \left(\frac{1}{384} X_{6}\right), \quad a_{7}=\exp \left(\frac{1}{4} X_{7}\right) .
\end{gathered}
$$


Let $\mathfrak{T}$ be the automorphism of $G$, whose differential $\mathfrak{T}_{*}: \mathfrak{g} \rightarrow \mathfrak{g}$ satisfies

$$
\begin{gathered}
\mathfrak{T}_{*}\left(X_{1}\right)=X_{1}, \quad \mathfrak{T}_{*}\left(X_{2}\right)=-X_{2}, \quad \mathfrak{T}_{*}\left(X_{3}\right)=-X_{3}, \quad \mathfrak{T}_{*}\left(X_{4}\right)=-X_{4}, \\
\mathfrak{T}_{*}\left(X_{5}\right)=-X_{5}, \quad \mathfrak{T}_{*}\left(X_{6}\right)=-X_{6}, \quad \mathfrak{T}_{*}\left(X_{7}\right)=X_{7} .
\end{gathered}
$$

Let $\alpha \in \operatorname{Aff} G$ be the element $\left(\exp \left(\frac{1}{2} X_{1}\right), \mathfrak{T}\right)$. The subgroup $E$ of Aff $G$ generated by $a_{1}, \ldots, a_{7}$ and $\alpha$ has a presentation of the form

$$
\begin{array}{rll}
E=\left\langle a_{1}, a_{2}, \ldots, a_{7}, \alpha\right. & {\left[a_{1}, a_{2}\right]=a_{3}^{2} a_{4}^{-4} a_{5}^{16} a_{6}^{112} a_{7}^{2},} & {\left[a_{3}, a_{4}\right]=1,} \\
& {\left[a_{1}, a_{3}\right]=a_{4}^{4} a_{5}^{-12} a_{6}^{32},} & {\left[a_{3}, a_{5}\right]=1,} \\
& {\left[a_{1}, a_{4}\right]=a_{5}^{6} a_{6}^{-24},} & {\left[a_{3}, a_{6}\right]=1,} \\
& {\left[a_{1}, a_{5}\right]=a_{6}^{8},} & {\left[a_{3}, a_{7}\right]=a_{6}^{-48},} \\
& {\left[a_{1}, a_{6}\right]=1,} & {\left[a_{4}, a_{5}\right]=1,} \\
& {\left[a_{1}, a_{7}\right]=1,} & {\left[a_{4}, a_{6}\right]=1,} \\
& {\left[a_{2}, a_{3}\right]=a_{5}^{-12} a_{6}^{-144} a_{7}^{-2},} & {\left[a_{4}, a_{7}\right]=1,} \\
& {\left[a_{2}, a_{4}\right]=1,} & {\left[a_{5}, a_{6}\right]=1,} \\
& {\left[a_{2}, a_{5}\right]=1,} & {\left[a_{5}, a_{7}\right]=1,} \\
& {\left[a_{2}, a_{6}\right]=1,} & {\left[a_{6}, a_{7}\right]=1,} \\
& {\left[a_{2}, a_{7}\right]=a_{5}^{-12} a_{6}^{-96},} & \\
& \alpha^{2}=a_{1}, & \\
& \alpha a_{2}=a_{2}^{-1} \alpha a_{3} a_{4}^{-1} a_{5}^{5} a_{6}^{47} a_{7}, & \alpha a_{3}=a_{3}^{-1} \alpha a_{4}^{2} a_{5}^{-3} a_{6}^{4}, \\
& \alpha a_{4}=a_{4}^{-1} \alpha a_{5}^{3} a_{6}^{-6}, & \alpha a_{5}=a_{5}^{-1} \alpha a_{6}^{4}, \\
& \alpha a_{6}=a_{6}^{-1} \alpha, & \alpha a_{7}=a_{7} \alpha
\end{array}
$$

This group $E$ is an almost-crystallographic subgroup of $\operatorname{Aff} G$. Moreover, $E$ is torsion-free and hence it is the fundamental group of an infranilmanifold $M_{1}$. The holonomy group is $\mathbb{Z}_{2}$ and the infranilmanifold is nonorientable. Note that this also implies that $M_{1}$ does not satisfy the condition in Theorem 3.1.

Now consider any self-map $f$ of $M_{1}$ inducing an endomorphism $\theta$ on the fundamental group $\pi_{1}\left(M_{1}\right)=E$ and having homotopy lift $(\delta, \mathfrak{D})$. We can distinguish two possibilities for $\mathfrak{D}_{*}$, namely $\mathfrak{D}_{*} \mathfrak{T}_{*}=\mathfrak{T}_{*} \mathfrak{D}_{*}$ or $\mathfrak{D}_{*} \mathfrak{T}_{*}=\mathfrak{D}_{*}$.

Any endomorphism $\mathfrak{D}_{*}$ of $\mathfrak{g}$ is completely determined by the images of its generators $X_{1}$ and $X_{2}$. We use the notation

$$
\begin{aligned}
& \mathfrak{D}_{*}\left(X_{1}\right)=\alpha_{1} X_{1}+\alpha_{2} X_{2}+\cdots+\alpha_{7} X_{7}, \\
& \mathfrak{D}_{*}\left(X_{2}\right)=\beta_{1} X_{1}+\beta_{2} X_{2}+\cdots+\beta_{7} X_{7} .
\end{aligned}
$$

Case 1: $\mathfrak{D}_{*} \mathfrak{T}_{*}=\mathfrak{T}_{*} \mathfrak{D}_{*}$. Here all the parameters $\alpha_{2}, \alpha_{3}, \alpha_{4}, \alpha_{5}, \alpha_{6}, \beta_{1}, \beta_{7}$ must be zero. Moreover, this case happens exactly when $\theta(\alpha)=a_{1}^{k_{1}} a_{2}^{k_{2}} \ldots a_{7}^{k_{7}} \alpha$ for some 
integers $k_{i} \in \mathbb{Z}$. It follows that

$$
\theta\left(a_{1}\right)=\theta(\alpha)^{2}=a_{1}^{2 k_{1}+1} a_{2}^{l_{2}} \cdots a_{7}^{l_{7}}
$$

for integers $l_{i} \in \mathbb{Z}$. This implies that $\alpha_{1}=2 k_{1}+1 \neq 0$. Thus in this case $\mathfrak{D}_{*}$ satisfies

$$
\mathfrak{D}_{*}\left(X_{1}\right)=\left(2 k_{1}+1\right) X_{1}+\alpha_{7} X_{7} \text { and } \mathfrak{D}_{*}\left(X_{2}\right)=\beta_{2} X_{2}+\beta_{3} X_{3}+\cdots+\beta_{6} X_{6} .
$$

If we now require that $\mathfrak{D}_{*}$ actually determine an endomorphism of $\mathfrak{g}$, there are 3 subcases to consider:

- $\beta_{2}=\beta_{3}=0$, in which case $\mathfrak{D}_{*}$ has a matrix representation, with respect to the basis $X_{1}, X_{2}, \ldots, X_{7}$ of the form

$$
\left(\begin{array}{ccccccc}
2 k_{1}+1 & 0 & 0 & 0 & 0 & 0 & 0 \\
* & 0 & 0 & 0 & 0 & 0 & 0 \\
* & 0 & 0 & 0 & 0 & 0 & 0 \\
* & 0 & 0 & 0 & 0 & 0 & 0 \\
* & * & * & 0 & 0 & 0 & 0 \\
* & * & * & * & 0 & 0 & 0 \\
* & * & * & * & 0 & 0 & 0
\end{array}\right) .
$$

Thus $\operatorname{det}\left(I_{7}-\mathfrak{D}_{*}\right)=\operatorname{det}\left(I_{7}-\mathfrak{T}_{*} \mathfrak{D}_{*}\right)=-2 k_{1}$ in this case.

- Another possibility is that $\beta_{2}=\alpha_{1}=1$. Now $\mathfrak{D}_{*}$ is of the form

$$
\left(\begin{array}{lllllll}
1 & 0 & 0 & 0 & 0 & 0 & 0 \\
* & 1 & 0 & 0 & 0 & 0 & 0 \\
* & * & 1 & 0 & 0 & 0 & 0 \\
* & * & * & 1 & 0 & 0 & 0 \\
* & * & * & * & 1 & 0 & 0 \\
* & * & * & * & * & 1 & 0 \\
* & * & * & * & * & * & 1
\end{array}\right)
$$

which again implies that $\operatorname{det}\left(I_{7}-\mathfrak{D}_{*}\right)=\operatorname{det}\left(I_{7}-\mathfrak{T}_{*} \mathfrak{D}_{*}\right)=0$.

- Finally it is possible that $\alpha_{1}=1$ and $\beta_{2}=-1$. Now $\mathfrak{D}_{*}$ is of the form

$$
\left(\begin{array}{rrrrrrr}
1 & 0 & 0 & 0 & 0 & 0 & 0 \\
* & -1 & 0 & 0 & 0 & 0 & 0 \\
* & * & -1 & 0 & 0 & 0 & 0 \\
* & * & * & -1 & 0 & 0 & 0 \\
* & * & * & * & -1 & 0 & 0 \\
* & * & * & * & * & -1 & 0 \\
* & * & * & * & * & * & 1
\end{array}\right),
$$


so again $\operatorname{det}\left(I_{7}-\mathfrak{D}_{*}\right)=\operatorname{det}\left(I_{7}-\mathfrak{T}_{*} \mathfrak{D}_{*}\right)=0$.

Case $2: \mathfrak{D}_{*} \mathfrak{T}_{*}=\mathfrak{D}_{*}$. This case forces $\beta_{1}=\beta_{2}=\cdots=\beta_{7}=0$. Hence $\mathfrak{D}_{*}$ is of the form

$$
\left(\begin{array}{lllllll}
k_{1} & 0 & 0 & 0 & 0 & 0 & 0 \\
k_{2} & 0 & 0 & 0 & 0 & 0 & 0 \\
k_{3} & 0 & 0 & 0 & 0 & 0 & 0 \\
k_{4} & 0 & 0 & 0 & 0 & 0 & 0 \\
k_{5} & 0 & 0 & 0 & 0 & 0 & 0 \\
k_{6} & 0 & 0 & 0 & 0 & 0 & 0 \\
k_{7} & 0 & 0 & 0 & 0 & 0 & 0
\end{array}\right) .
$$

Again we obtain that $\operatorname{det}\left(I_{7}-\mathfrak{D}_{*}\right)=\operatorname{det}\left(I_{7}-\mathfrak{T}_{*} \mathfrak{D}_{*}\right)=1-k_{1}$.

In either case, we can use Theorem 2.3 as before to conclude that Anosov's theorem holds on this infranilmanifold.

\section{References}

[Anosov 1985] D. V. Anosov, "Nielsen numbers of mappings of nil-manifolds", Uspekhi Mat. Nauk 40:4 (1985), 133-134. In Russian; translated in Russian Math. Surveys 40:4 (1985), 149-150. MR 87c:55001 Zbl 0594.55002

[Brown 1971] R. F. Brown, The Lefschetz fixed point theorem, Scott, Foresman, Glenview, IL, 1971. MR 44 \#1023 Zbl 0216.19601

[Brown 1982] K. S. Brown, Cohomology of groups, Graduate Texts in Mathematics 87, Springer, New York, 1982. MR 83k:20002 Zbl 0584.20036

[Dekimpe 1996] K. Dekimpe, Almost-Bieberbach groups: affine and polynomial structures, Lecture Notes in Mathematics 1639, Springer, Berlin, 1996. MR 2000b:20066 Zbl 0865.20001

[Dekimpe et al. 2004] K. Dekimpe, B. De Rock, and W. Malfait, "The Anosov theorem for flat generalized Hantzsche-Wendt manifolds”, J. Geom. Phys. 52:2 (2004), 174-185. MR 2005f:37047 Zbl 1068.37010

[Dekimpe et al. 2006] K. Dekimpe, B. De Rock, and H. Pouseele, "The Anosov theorem for infranilmanifolds with an odd order abelian holonomy group", Fixed Point Th. Appl. Article ID 63939 (2006).

[Jiang 1983] B. J. Jiang, Lectures on Nielsen fixed point theory, Contemporary Mathematics 14, American Mathematical Society, Providence, R.I., 1983. MR 84f:55002 Zbl 0512.55003

[Jiang 1993] B. J. Jiang, "Commutativity and Wecken properties for fixed points on surfaces and 3-manifolds", Topology Appl. 53:2 (1993), 221-228. MR 95c:55003 Zbl 0791.55002

[Keppelmann and McCord 1995] E. C. Keppelmann and C. K. McCord, "The Anosov theorem for exponential solvmanifolds", Pacific J. Math. 170 (1995), 143-159. MR 96j:55005 Zbl 0856.55003

[Kiang 1989] Kiang T.-h., The theory of fixed point classes, Springer, Berlin and New York, 1989. MR 90h:55002 Zbl 0676.55001

[Lee 1995] K. B. Lee, "Maps on infra-nilmanifolds", Pacific J. Math. 168:1 (1995), 157-166. MR 96k:57041 Zbl 0920.55003 
[Porteous 1972] H. L. Porteous, "Anosov diffeomorphisms of flat manifolds", Topology 11 (1972), 307-315. MR 45 \#6035 Zbl 0237.58015

[Wecken 1942] F. Wecken, "Fixpunktklassen, III: Mindestzahlen von Fixpunkten”, Math. Ann. 118 (1942), 544-577. MR 5,275b JFM 0027.26503

Received May 2, 2005.

KAREL DEKIMPE

DEPARTMENT OF MATHEMATICS

KATHOLIEKE UNIVERSITEIT LEUVEN CAMPUS KORTRIJK

UNIVERSITAIRE CAMPUS

ETIENNE SABBELAAN 53

B-8500 KORTRIJK

BELGIUM

Karel.Dekimpe@kuleuven-kortrijk.be

BRAM DE ROCK

DEPARTMENT OF MATHEMATICS

KATHOLIEKE UNIVERSITEIT LEUVEN CAMPUS KORTRIJK

UNIVERSITAIRE CAMPUS

ETIENNE SABBELAAN 53

B-8500 KORTRIJK

BELGIUM

Bram.DeRock@kuleuven-kortrijk.be

WiM MALFAIT

DEPARTMENT OF MATHEMATICS

KATHOLIEKE UNIVERSITEIT LEUVEN CAMPUS KORTRIJK

UNIVERSITAIRE CAMPUS

ETIENNE SABBELAAN 53

B-8500 KORTRIJK

BELGIUM

Wim.Malfait@kuleuven-kortrijk.be 